\title{
FIRST RECORD OF CANTHARELLUS MINOR IN VIETNAM
}

\author{
Hung Huu Phan ${ }^{1, ~}$, Thuan Duc Lao², Thuy Huyen Ai Le ${ }^{2}$, Khanh Quoc Hoang ${ }^{3}$, Nguyen Binh \\ Truong ${ }^{4}$, Giang Thi Lam Ngo 5 \\ ${ }^{I}$ Tay Nguyen Institute of Scientific Research, Vietnam Academy of Science and Technology \\ ${ }^{2}$ Ho Chi Minh City Open University, Ho Chi Minh City \\ ${ }^{3}$ Institute of Tropical Biology, Vietnam Academy of Science and Technology \\ ${ }^{4}$ Da Lat University, Da Lat \\ ${ }^{5}$ NTT Institute of Hi-Technology, Nguyen Tat Thanh University, Ho Chi Minh City
}

$\bowtie$ To whom correspondence should be addressed. E-mail: huuhung_0478@yahoo.com

Received: 02.11.2017

Accepted: 28.12.2017

\section{SUMMARY}

This species of mushroom with orange fruiting bodies and yellow flesh grows in clumps on the forest land in the coordinates $11^{\circ} 56^{\prime} 34.45^{\prime \prime} \mathrm{N}, 108^{\circ} 28^{\prime} 33.56^{\prime \prime} \mathrm{E}$ in the pine (Pinus kesiya) forest, Da Lat City, Lam Dong Province, Vietnam. The results of analysis on the morphology, both macro- and micro-morphological characteristics of this mushroom showed that, pileus: $5-15 \mathrm{~mm}$ wide, infundibuliform; yellowish to orange; margin incurred, wavy-liked margin, non-striate; Pileus surface: smooth, scaleless, yellowish; Lamellae: distant, decurrent, not intervenose, concolorous to pileus; Context: concolourous to the pileus, slight sweetness, aromatic flavor; Stipe: cylindrical shape, surface smooth, concolourous to pileus, 1-2 mm diameter, 20-50 mm length; Basidiospores: 6-11.5 x 4-6.5 $\mu \mathrm{m}$, ovoid-ellipsoid with smooth surface; Basidia: 65 x $10 \mu \mathrm{m}$, cornuted 4-6 spores per basidium. Phylogenetic analysis of $n r L S U$ sequence yielded consistent topology in different taxa of Cantharellus. The phylogenetic position of XC02 was obtained and accepted at sub-generic level: subgenus Parvocantharellus. This clade was suggested to be monophyletic, and separated from other sub-generic levels. Morphologically phylogenetically distinct from the other species of clade 4, such as C. appalachiensis, $C$. tabernensis, C. aff. Congolensis. The highly supported monophyletic group with referent Cantharellus minor was obtained with the bootstrap value of 99 , indicated that $\mathrm{XC} 02$ was significant closely to Cantharellus minor. Phylogenetic of $n r L S U$ analysis revealed clades with statistical support corresponding to morphological observation, thus, XC2 was concluded as Cantharellus minor.

Keywords: Cantharellus, Cantharellus minor, nrLSU, funnel-shaped fungus,taxonomy

\section{INTRODUCTION}

Cantharellus minor Peck Rep. (Annual) New York State Mus. Nat. Hist. 23. 1872. was originally described by Peck (1872). This species, a popularly known and commercialized fungus, belongs to the genus Cantharellus Adans.: Fr. $C$. minor has been reported as one of the smallest of the Cantharellus, found on soil, forming ectomycorrhizal association with the tree of Cedrus deodara, Quercus dilatata, etc. The yellowish lamellae are described as very narrow, distant, sparing branched, decurrent, concolourous, and fade to yellowish white in maturity. The pileus range from $0.5 \mathrm{~cm}$ to $3.0 \mathrm{~cm}$ wide, thin, convex, expanded and depressed, becoming funnel-shaped in some. The stipe is less than $4 \mathrm{~cm}$, base attenuated, central, solid, concolours to the pileus, surface glabrous. They fruit in the summer and fall (Peck, 1872; Kuo, 2006). This species is native to Eastern North America, Canada, Western Ghats, Kerala, India, Japan, etc., however, it was not recorded in Vietnam. During the survey of fungi in Lam Dong $\left(11^{\circ} 56^{\prime} 34.45^{\prime \prime} \mathrm{N}, 108^{\circ} 28^{\prime} 33.56^{\prime \prime} \mathrm{E}\right)$, a province located in the Central highlands (namely Tay Nguyen) region of Vietnam, specimens belonging to Cantharellus were collected. In this study, these Vietnamese specimens were identified based on morphology, molecular phylogenetic analysis and compatibility between our collected specimens and other strains in many countries of the world. 


\section{MATERIALS AND METHODS}

\section{Fungal collection}

Basidiomata, coded XC02, was collected in a Pine forest (Pinus kesiya Royle ex Gordon), a fastgrowing, has natural distribution in South-East Asia, ca. $1502 \mathrm{~m}$ altitude, at Xuan Tho Commune, Da Lat City, Lam Dong Province, Vietnam. General information on collection: Height: $1.502 \mathrm{~m}$; Humidity: $87 \%$; Temperature: $20^{\circ} \mathrm{C}$; Light intensity: 3.012 lux; Coordinates: $11^{\circ} 56^{\prime} 34.45^{\prime \prime} \quad \mathrm{N}$, $108^{\circ} 28^{\prime} 33.56^{\prime \prime}$ E. The specimens were pick up by digging them out carefully and preserved by immediately wrapped in the wax paper and placed in the collection bags. Attempts were made to collect all different developing stages of the basidiocraps to have an idea of size, color and shapes. In the laboratory, specimens were exposed to room temperature, and then, $1 \%$ Mercury (II) chloride was impregnated for $5-10$ minutes, finally, dried at $60^{\circ} \mathrm{C}$ and stored for further analysis.

\section{Macro- and micromorphology analysis}

Morphological observations were studied and recorded by using guidelines according to Lodge et al., (2004). Macroscopic characters were carefully recorded in the field keys specially designed for the purpose and photographed using digital camera. The following macroscopic characters including pileus, lamellae, context, etc., were also noted. The color notation was noted from Kornerup, Wanscher (1981). Micromorphological features of specimens were examined and observed with Olympus B51 (Tokyo, Japan) microscopes with light and phase contrast optics. The following microscopic characters were found particularly for the identification and confirmation of Cantharellaceae, including spore morphology, basidiospores, hyphae, basidium structure.

\section{Molecular studies: DNA extraction, PCR and DNA sequencing}

For molecular characterization, genomic DNA was isolated from the dried fruit bodies of collected specimens of Cantharellaceae. The dried fruit body's powder of specimen was added in a lysis buffer containing $10 \mathrm{mM}$ Tris- $\mathrm{HCl} \mathrm{pH}$ 8.0, $10 \mathrm{mM}$ EDTA, $150 \mathrm{mM} \mathrm{NaCl}, 2 \%$ SDS, $0.1 \mathrm{mg} / \mathrm{mL}$ Proteinase $\mathrm{K}$ ). During incubation at $65^{\circ} \mathrm{C}$ for overnight, the cell suspension was mixed thoroughly by inverting the tube several times. Then, the supernatant was collected by centrifugation. The solution of $700 \mu \mathrm{L}$ of PCI (Phenol/Chloroform/Isoamylalcohol with ratio of $25: 24: 1)$ was added and centrifuged. The upper solution was collected, precipitated with absolute ethanol, and washed with $70 \%$ ethanol. DNA concentration was identified by using $\mathrm{OD}_{260}$. The DNA was purified by elution through the manufacturer's instructions of Wizard®DNA cleanup system in order to remove the contaminants. Finally, isolated genomic DNA were kept in TE buffer at $-20^{\circ} \mathrm{C}$ for further studies.

Primer pairs: LR0R (Forward primer): 5'GTACCCGCTGAACTTAAGC-3' and LR5 (Reversed primer): 5'-ATCCTGAGGGAAACTTC3' (Vilgalys, Hester, 1990) were used to amplify a portion of $n r L S U$ (nuclear ribosomal large subunit) gene. The final volume for PCR was $15 \mu \mathrm{L}$ with a specified program: 1 cycle of $95^{\circ} \mathrm{C}$ for 5 mins; 40 cycles of $95^{\circ} \mathrm{C}$ in $30 \mathrm{~s}, 55^{\circ} \mathrm{C}$ in $30 \mathrm{~s} .72^{\circ} \mathrm{C}$ in 2 mins; 1 cycle of $72^{\circ} \mathrm{C}$ in 5 mins. Aliquots of amplification products $(5 \mu \mathrm{L})$ were electrophoresed on $2.0 \%$ agarose gel and visualized on UV transilluminator. The amplified product was sequenced at Nam Khoa (Vietnam) Company with the same primers.

\section{Sequence proofreading and phylogenetic analysis}

DNA sequences were proofread to remove ambiguous signals at both ends. The software, including SeaView 4.2.12, Chromas Lite 2.1.1, were used for proofreading. The data set of $n r L S U$ was established by sequences downloaded from GenBank. Phylogenetic tree was constructed with MEGA 6.0 with a 1000 replicate bootstrap based on the maximum parsimony method (Dunham et al., 2003).

\section{RESULTS}

\section{Taxonomy}

Cantharellus minor Peck Rep. (Annual) New York State Mus. Nat. Hist. 23. 1872.

[Description based on Vietnamese specimens, Fig. 1]. Pileus: 5-15 mm wide, infundibuliform; yellowish to orange; margin incurred, wavy-liked margin, non-striate. Pileus surface: smooth, scaleless, yellowish; Lamellae: distant, decurrent, not intervenose, concolorous to pileus; Context: concolourous to the pileus, slight sweetness, aromatic flavor. Stipe: cylindrical shape, surface smooth, concolourous to pileus, 1-2 mm diameter, 20-50 mm length. Basidiospores: 6-11.5 x 4-6.5 $\mu \mathrm{m}$, ovoid-ellipsoid with smooth surface. Basidia: $65 \mathrm{x}$ $10 \mu \mathrm{m}$, cornuted 4-6 spores per basidium. 


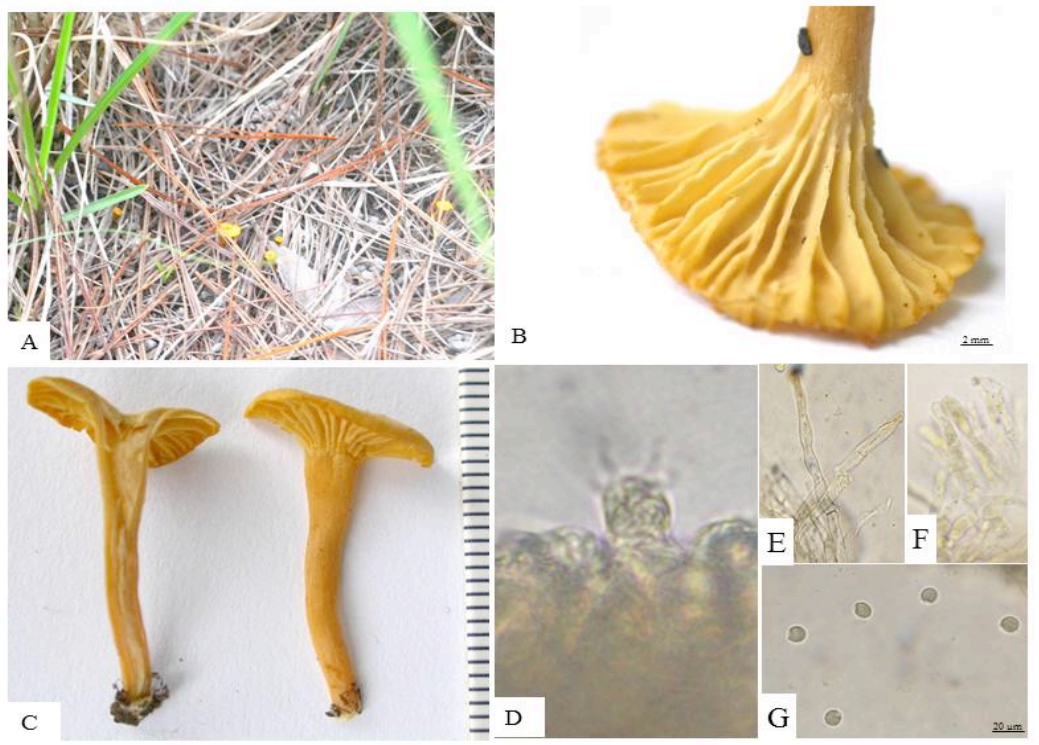

Figure 1. A. Cantharellus minor collected in Pine forest; B. Lamellae; C. Fruit body; D. Basidia; E-F: Hyphae; G: Basiospore

\section{Amplification of $n r L S U$ gene, phylogenetic analysis of $n r L S U$ data set}

DNA after extraction and purification was amplified with primer LR0R and LR5, then, electrophoresis on $2.0 \%$ agarose gel showed a significant and clear band of 950 bps (Fig. 2A). The
PCR products was sequenced, as the results, the signals of peaks in both strands were significant and good for reading (Fig. 2B). According to BLAST results, $\mathrm{XC02}$ sequence was similar to $C$. minor, strain BB 07.002 (Accession number: KF294625) with total score $=\max$ score $=1544$, Ident $=99 \%$, Evalue $=0.0$ )
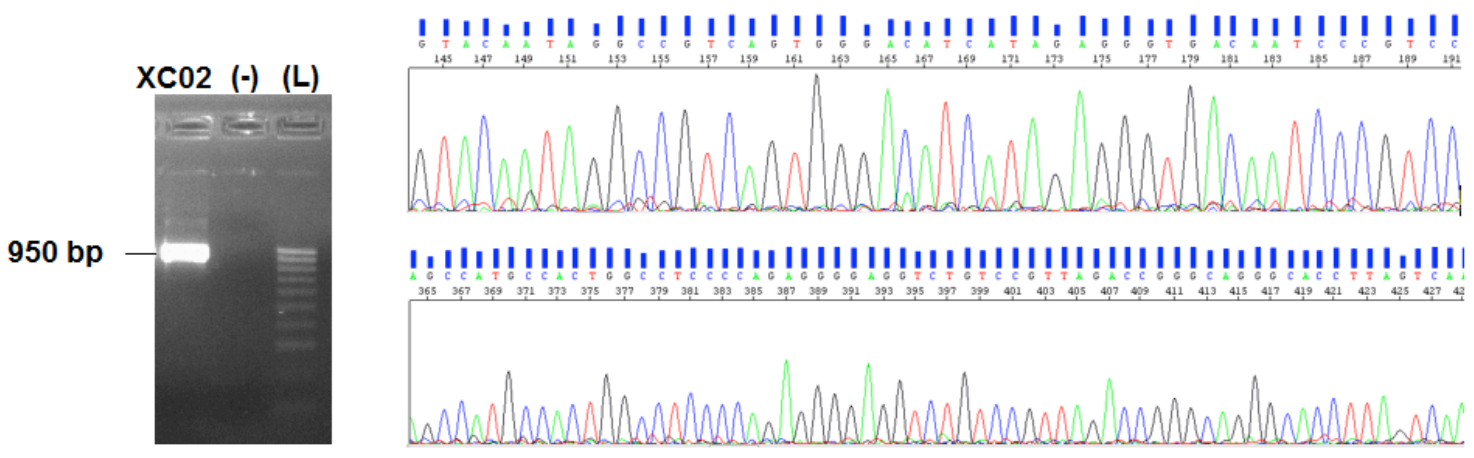

Figure 2. (A) Electrophoresis of PCR product of $n r L S U$. (L) 100 bp Ladder, (-) negative control, and XC002; (B) A part of the sequences from the forward primer (upper) and reverse primer (lower).

The final data set of $n r L S U$ consisted of 61 sequences of 633 characters, including 58 sequences belonging to Cantharellus (reference data), 1 sequence belonged to Craterellus (served as outgroup), and a XC02 sequence. The molecular phylogenetic analysis based on the topology constructed by maximum parsimony, showed the formation outgroup
(Craterellus) and six clades that are here obtained and accepted at sub-generic levels, including subgenus Cantharellus (Clade 1), subgenus Rubrinus (Clade 2), subgenus Cinnabarinus (Clade 3), subgenus Parvocantharellus (Clade 4), subgenus Pseudocantharellus (Clade 5), and subgenus Afrocantharellus (Clade 6). 
According to $\mathrm{XC} 02$, formed the monophyletic group with several Clade $4 \mathrm{~b}$ (belonging to subgenus Parvocantharellus) referent sequences, including $C$. appalachiensis, C. tabernensis and C. minor. Notably, within this clade, $\mathrm{XC} 02$ formed a higly supported monophyletic group (Bootstrap = 99) with two sequences: C. minor (Accession numbers: KF294625, KF294632) (Fig. 3), and separated this group from other referent taxon in clade 4. Combining with morphological identification, the molecular identification showed similar result as C. minor. Therefore, we concluded that $\mathrm{XC} 02$ is $C$. minor.

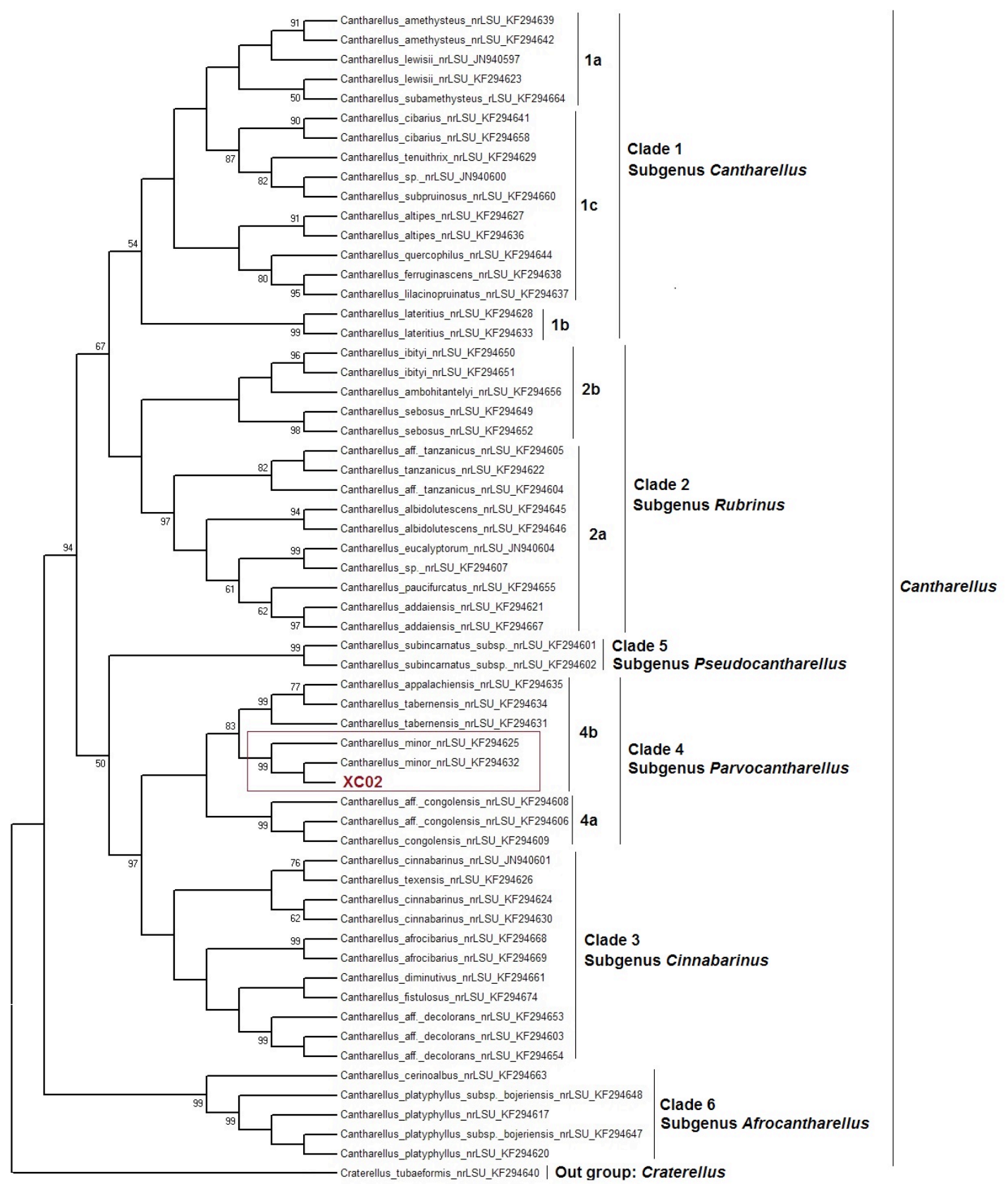

Figure 3. Molecular phylogenetic analysis of $n r L S U$ by maximum parsimony for 61 sequences with a 1000 replicate bootstrap proportions. 


\section{Discussion}

The traditional taxonomy of fungi emphasizes the morphology, including marco-, and mircromorphology features to delimit a taxon. Its taxonomy relies on the size, shape of fruiting structures, lamellae, basidium, spore morphology, coloration and habitat to define taxa. In this study, the description of the morphology of $\mathrm{XC02}$ with numerous striking features showed the similar to the morphological description of Cantharellus minor Peck Rep. (Annual) New York State Mus. Nat. Hist. 23. 1872. which was originally described by Peck (1872).According to Mitchell et al., (1995), they suggested that molecular phylogenetic approaches to fungal evolution have proved valuable information towards the goal of understanding the relationship among specific fungal group. In this study, $n r L S U$ (the nuclear ribosomal large subunits), the most popular locus for DNA-based mycological studies for taxon identification, was used. The $n r L S U$ is a part of the rDNA gene for the nuclear genome, has been widely used as the potential marker for fungal species identification in recent years. Noteworthy taxonomic works on the nrLSU phylogenetics analysis on Cantharellaceae include those of Dahlman et al., (1993), Feibelman et al., (1997), Moncalvo et al., (2006), Arora and Dunham, (2008). According to phylogenetic analysis, phylogenetic analysis of $n r L S U$ sequence yielded consistent topology in different taxa of Cantharellus. The phylogenetic position of $\mathrm{XC02}$ was here obtained and accepted at sub-generic level: subgenus Parvocantharellus. This clade was suggested to be monophyletic, and separated from other sub-generic levels. Morphologically phylogenetically distinct from the other species of clade 4, such as $C$. appalachiensis, C. tabernensis, C. aff. Congolensis. The highly supported monophyletic group with referent $C$. minor was obtained with the bootstrap value of 99 , indicated that $\mathrm{XC02}$ was significant closely to $C$. minor. In conclusion, phylogenetic of $n r L S U$ analysis revealed clades with statistical support corresponding to morphological observation, thus, $\mathrm{XC} 2$ was concluded as $C$. minor.

\section{CONCLUSION}

We have successfully applied the morphological characterization in combination with phylogenetic analysis of $n r L S U$ to delimit sample $\mathrm{XC02, \text {which }}$ collected in a Pine forest (Pinus kesiya Royle ex Gordon), ca. 1500 altitude, at Xuan Tho Commune, Da Lat City, Lam Dong Province, Vietnam and identified as Cantharellus minor Peck. This is the first record of Cantharellus minor in Vietnam.

Acknowledgments: We express our special thanks to Tay Nguyen Institute of Scientific Research, Faculty of Biology, Da Lat University and Faculty of Biotechnology, Ho Chi Minh City Open University for the genuine support throughout this research work.

\section{REFERENCES}

Dunham SM, O' Dell TE, Molina R (2003) Analysis of nrDNA sequences and microsatellite allele frequencies reveals a cryptic chanterelle species Cantharellus cascadensis sp. nov. from the American Paci fi c Northwest. Mycol Res 107:1163-1177.

Kornerup A, Wanscher JH (1981) Methuen handbook of colour, 3rd edition. 1981. Eyre Methuen, London, UK.

Kuo M (2006) Cantharellus minor. MushroomExpert.Com. Retrieved 2011-03-24.

Lodge DJ, Joseph F, Ammirati, Thomas E, Dell O, Gregory M, Mueller (2004) Collecting and describing macrofungid. Science and Technology Rights Department in Oxford, UK: 128-158.

Mitchell JI, Roberts PJ, Moss ST (1995) Sequence or Structure. A short review on the application of nucleic acid sequence information to fungal taxonomy. Mycologist 9: $67-76$

Moncalvo JM, Nilsson RH, Koster B, Dunham SM, Bernauer T, Matheny PB, Porter TM, Margaritescu S, Weiss M, Garnica S, Danell E, Langer G, Langer E, Larsson E, Larsson KH, Vilgalys R (2006) The cantharelloid clade: dealing with incongruent gene trees and phylogenetic reconstruction methods 98(6): 937-948.

Peck CH (1872) Report of the Botanist (1869). Annual Report on the New York State Museum of Natural History 23: $27-135$.

Vilgalys R, Hester M (1990) Rapid genetic identification and mapping of enzymatically amplified ribosomal DNA from several Cryptococcus species. Journal of bacteriology 172(8): 4238-4246. 